\title{
NK-1 Receptor Immunoreactivity in Distinct Morphological Types of Lamina I Neurons of the Primate Spinal Cord
}

\author{
X. H. Yu, ${ }^{1}$ E.-T. Zhang, ${ }^{3}$ A. D. Craig, ${ }^{3}$ R. Shigemoto, ${ }^{4}$ A. Ribeiro-da-Silva, ${ }^{1,2}$ and Y. De Koninck ${ }^{1}$ \\ ${ }^{1}$ Department of Pharmacology and Therapeutics, and ${ }^{2}$ Anatomy and Cell Biology, McGill University, Montréal, Quebec, \\ H3G 1 Y6 Canada, ${ }^{3}$ Division of Neurobiology, Barrow Neurological Institute, Phoenix. Arizona 85013, and ${ }^{4}$ National \\ Institute for Physiological Sciences, Okazaki 444-8585, Japan
}

In cat and monkey, lamina I cells can be classified into three basic morphological types (fusiform, pyramidal, and multipolar), and recent intracellular labeling evidence in the cat indicates that fusiform and multipolar lamina I cells are two different types of nociceptive cells, whereas pyramidal cells are innocuous thermoreceptive-specific. Because earlier observations indicated that only nociceptive dorsal horn neurons respond to substance $\mathrm{P}$ (SP), we examined which morphological types of lamina I neurons express receptors for SP (NK-1r).

We categorized NK-1r-immunoreactive (IR) lamina I neurons in serial horizontal sections from the cervical and lumbar enlargements of four monkeys. Consistent results were obtained by two independent teams of observers. Nearly all NK-1r-IR cells were fusiform (42\%) or multipolar (43\%), but only $6 \%$ were pyramidal (with $9 \%$ unclassified). We obtained similar findings in three monkeys in which we used double-labeling immuno-

Lamina I, the marginal layer in the superficial dorsal horn of the spinal cord (Rexed, 1952), is an integral component of the central representation of pain and temperature sensibility (Perl, 1984; Willis, 1985; Light, 1992; Craig, 1996b). It provides a major output pathway from the spinal cord to higher structures (brainstem and thalamus) that carries activity from small diameter afferents (A $\delta$ and $\mathrm{C}$ fibers) from most tissues of the body (Craig, 1996a). The complex integration of these types of inputs within lamina $\mathrm{I}$ is associated with a rich concentration of neuropeptides (Ribeiro-da-Silva and Cuello, 1995). Although numerous studies have highlighted dense peptidergic input to lamina I, few have provided detailed information on peptidergic innervation or the expression of their receptors in relation to defined neurons (e.g., Priestley and Cuello, 1989; De Koninck et al., 1992; Brown et al., 1995; Li et al., 1996, 1997; Ma et al., 1996; Marshall et al., 1996; Naim et al., 1997).

Emerging criteria for the identification of lamina I neurons on the basis of their structural and functional features provide a method for the phenotypic characterization of specific cell populations. Studies with the Golgi technique in the cat (Gobel, 1978)

\footnotetext{
Received Dec. 16, 1998; revised Feb. 12, 1999; accepted Feb. 16, 1999.

This study was supported by National Institute of Health Grants NS 34022 to Y.D.K. and NS 25616 to A.D.C., by Canadian Medical Research Council (MRC) Grants MT 12942 to Y.D.K. and MT 12170 to A.R.S., and by the Barrow Neurological Foundation. Y.D.K. is a Scholar of the Canadian MRC. We thank A. Constantin and A. Forster for expert technical assistance and Dr. M. Wikstrom for generously supplying monoclonal antibodies against CTb.

Correspondence should be addressed to Dr. Yves De Koninck, Department of Pharmacology and Therapeutics, McGill University, 3655 Drummond, Montreal, Quebec, H3G 1Y6 Canada.

Copyright (C) 1999 Society for Neuroscience $\quad 0270-6474 / 99 / 193545-11 \$ 05.00 / 0$
}

cytochemistry to identify NK-1r-IR and spinothalamic lamina I neurons retrogradely labeled with cholera toxin subunit $b$ from the thalamus; most NK-1r-IR lamina I spinothalamic neurons were fusiform (48\%) or multipolar (33\%), and only $10 \%$ were pyramidal. In contrast, most $(\sim 75 \%)$ pyramidal and some $(\sim 25 \%)$ fusiform and multipolar lamina I spinothalamic neurons did not display NK-1r immunoreactivity.

These data indicate that most fusiform and multipolar lamina I neurons in the monkey can express NK-1r, consistent with the idea that both types are nociceptive, whereas only a small proportion of lamina I pyramidal cells express this receptor, consistent with the previous finding that they are nonnociceptive. However, these findings also indicate that not all nociceptive lamina I neurons express receptors for SP.

Key words: dorsal horn; spinothalamic; nociception; substance $P$; monkey; functional specialization

and the rat (Lima and Coimbra, 1986) first demonstrated that several morphological types of lamina I cells can be differentiated according to cell body shape and the characteristics of proximal dendritic branches. Although other descriptions were attempted in the monkey (Willis et al., 1979; Beal et al., 1981), recent retrograde labeling studies have provided consistent evidence in both the cat (Zhang et al., 1996) and the monkey (Zhang and Craig, 1997) that lamina I spinothalamic neurons can be grouped under the basic categories of fusiform $(\mathrm{F})$, pyramidal $(\mathrm{P})$, and multipolar (M). These three morphological types are relatively evenly distributed in the cervical and lumbar enlargements (Lima and Coimbra, 1986; Zhang et al., 1996; Zhang and Craig, 1997). Recent intracellular labeling evidence has revealed a direct correspondence between the morphological characteristics of lamina I neurons and their functional responses to cutaneous stimulation in the cat (Han et al., 1998). Fusiform cells appear to be nociceptive-specific neurons (NS), responsive only to noxious heat and pinch, whereas multipolar cells are polymodal nociceptive neurons (HPC) responsive to noxious heat, pinch, and cold, and pyramidal cells are innocuous thermoreceptive neurons (COLD), responsive only to cooling (Craig and Kniffki, 1985; Craig and Bushnell, 1994; Dostrovsky and Craig, 1996; Han et al., 1998). These results are supported by findings from Golgi studies that fusiform cells have unmyelinated axons, but pyramidal and multipolar cells have myelinated axons (Gobel, 1978; Lima and Coimbra, 1986) because NS cells have slow conduction velocities, whereas HPC and COLD cells have faster conduction velocities (Craig and Kniffki, 1985; Craig and Serrano, 1994). Recent observations suggest that this structure-function correspondence 
may also be present in the monkey (Craig et al., 1999). This possibility provides a basis to test for further phenotypic specialization of lamina I cells in the primate.

Of the abundant peptidergic input to the superficial dorsal horn, the neurokinin substance P (SP) is the best characterized and has been mainly associated with nociception (for review, see Henry, 1982; Cuello, 1987; Otsuka and Yanagisawa, 1990). In particular, SP-mediated excitation via activation of NK-1 receptors (NK-1r) is restricted to nociceptive neurons (Henry, 1976; Randic and Miletic, 1977; Salter and Henry, 1991). Additional evidence indicates that NK-1r-mediated synaptic responses to endogenous SP released by activation of nociceptive afferent fibers (De Koninck and Henry, 1991) occur only in nociceptive neurons (De Koninck et al., 1992), and that non-nociceptive mechanoreceptive neurons do not receive significant amounts of direct SP input (De Koninck et al., 1992; Ma et al., 1996). Furthermore, innocuous cooling-sensitive primary afferent c-fibers do not appear to express SP (Lawson et al., 1997). Thus, these findings suggest that non-nociceptive neurons may generally not express NK-1r, the SP receptor.

We therefore examined whether the distinct morphological types of lamina I neurons differentially express NK-1r in adult monkeys. In addition, we quantified NK-1r immunoreactivity in retrogradely labeled spinothalamic tract (STT) neurons using thalamic injections of cholera toxin subunit b $(\mathrm{CTb})$ (Ericson and Blomqvist, 1988; Zhang and Craig, 1997).

\section{MATERIALS AND METHODS}

Three cynomolgus monkeys (Macaca fascicularis) and one rhesus monkey (Macaca mulatta) were used for single-labeling experiments of NK-1r immunoreactivity in lamina I. The animals were deeply anesthetized with sodium pentobarbital, then perfused transcardially with $4 \%$ paraformaldehyde in $0.1 \mathrm{M}$ phosphate buffer (PB), $\mathrm{pH} 7.4$, for at least 30 min. The whole spinal cord was removed and post-fixed in $4 \%$ paraformaldehyde for $2 \mathrm{hr}$. Finally, the tissue was transferred to $30 \%$ sucrose in $\mathrm{PB}$ and stored at $4^{\circ} \mathrm{C}$ for later immunocytochemical processing.

Three additional cynomolgus monkeys were used for retrograde labeling experiments combined with detection of NK-1r immunoreactivity. Retrograde labeling of STT neurons with subunit $\mathrm{b}$ of cholera toxin was performed as previously described (Zhang and Craig, 1997). Briefly, the animals were anesthetized with sodium pentobarbital $(40 \mathrm{mg} / \mathrm{kg}$, i.p., with intravenous supplements) and placed in a stereotaxic apparatus. Body temperature was maintained at $36^{\circ} \mathrm{C}$. Under aseptic conditions, a window in the skull was made to provide access to the left thalamus. The stereotaxic coordinates of the boundaries of the somatosensory thalamus were identified electrophysiologically by recording during several penetrations with a glass-coated tungsten microelectrode. Injection sites were chosen by extrapolation from this map to include nearly all of the dorsal thalamus, but particularly the major lamina I STT termination sites: the posterior part of the ventral medial nucleus (VMpo), the ventral posterior inferior nucleus (VPI), and the ventral caudal part of the medial dorsal nucleus (MDvc) (Craig et al., 1994). Eight injections of an aqueous solution of $0.4-1 \%$ CTb (Sigma, St. Louis, MO) were made using a Hamilton syringe for a total of 2-10 $\mu$ l. The needle was left in place for 5-15 min after each injection. The animals were allowed to survive for $21 \mathrm{~d}$. After the survival period, the monkeys were deeply anesthetized and perfused transcardially with a solution of $4 \%$ paraformaldehyde in $\mathrm{PB}$. The brain and spinal cord were removed and post-fixed in $4 \%$ paraformaldehyde in $\mathrm{PB}$ for $4 \mathrm{hr}$ and then transferred to $30 \%$ sucrose in $\mathrm{PB}$ and stored at $4^{\circ} \mathrm{C}$ for later immunocytochemical processing.

Immunocytochemistry. Serial $40-\mu \mathrm{m}$-thick sections were cut in the transverse and horizontal planes from segments of the cervical (C6-7) and lumbar (L6-7) enlargements. These segments were chosen because they contain a high density of lamina I cells and because the three morphological types of lamina I spinothalamic cells were found to be relatively equally represented at these levels (Zhang and Craig, 1997). Only these segments were used for quantitative analysis of NK-1r immunoreactivity. The sections were pretreated with $5 \%$ normal goat serum and then placed in a polyclonal antibody raised in rabbit against the NK-1r
(Shigemoto et al., 1993; Nakaya et al., 1994) for $48-72$ hr at $4^{\circ} \mathrm{C}$. After rinsing in $0.2 \%$ Triton X-100 in PBS (PBS-T), the sections were incubated for $2 \mathrm{hr}$ at room temperature in biotinylated goat anti-rabbit $\mathrm{IgG}$, rinsed again, and then incubated at room temperature with an $\mathrm{ABC}$ complex (Vector Laboratories, Burlingame, CA). After further washes, the sections were incubated with 3,3'-diaminobenzidine tetrahydrochloride and $\mathrm{H}_{2} \mathrm{O}_{2}$.

Double-labeling immunocytochemistry for the detection of STT and $N K-1 r-I R$ neurons. For experiments with retrograde labeling, serial 50$\mu \mathrm{m}$-thick sections were cut in the transverse plane through the thalamus. A one-in-three series of thalamic sections was stained with thionin, and all other sections were processed immunocytochemically with the ABC technique for $\mathrm{CTb}$ labeling using a mouse anti-CTb monoclonal antibody (see below) as previously described (Zhang et al., 1996).

For double labeling of NK-1r and CTb immunoreactivity, serial 40$\mu \mathrm{m}$-thick sections were cut in the transverse and horizontal planes of segments C6-7 and L6-7. Sections were incubated in 10\% normal donkey serum for $1 \mathrm{hr}$ and then for $48-72 \mathrm{hr}$ in a mixture of primary antibodies consisting of a rabbit anti-NK-1r polyclonal antibody and a mouse anti-CTb monoclonal antibody (a 1:1 mixture of antibodies from clones CT2 and CT9; courtesy of Dr. M. Wikstrom) at $4^{\circ} \mathrm{C}$. After several rinses in PBS-T, the sections were incubated in a mixture of sheep anti-rabbit IgG conjugated to FITC (ICN Biochemicals, Montréal, Québec, Canada) and biotinylated horse anti-mouse $\operatorname{IgG}$ (Vector) for $2 \mathrm{hr}$ at room temperature. After further rinses, the sections were incubated in streptavidin conjugated to Texas Red (Vector) for $2 \mathrm{hr}$. All antibodies were diluted in PBS-T containing 5\% normal donkey serum. Finally, the sections were washed, mounted on gelatin-subbed slides, and coverslipped with an anti-fading mounting medium (Dako, High Wycombe, UK).

Some sections were examined under a Zeiss LSM 410 confocal scanning laser microscope equipped with argon-krypton and helium-neon lasers and appropriate filter sets for independent detection of FITC and Texas Red. Superimposition of few (three to seven) serial optical sections was used to compare the nature and subcellular distribution of each of the labels.

Classification of neurons and quantification. Serial horizontal sections were examined under a light microscope using $10-40 \times$ objectives. All labeled lamina I neurons of each of the three main types, F, P, and M, were identified on the basis of somatal shape and dendritic orientation in horizontal sections following the classification scheme used earlier (Zhang et al., 1996; Zhang and Craig, 1997; Han et al., 1998). The horizontal plane of section was used for the classification and quantification because the perikarya and dendrites of lamina I cells are mainly oriented in the rostrocaudal and mediolateral axes, and most of their dendrites are confined to lamina I (Gobel, 1978; Light et al., 1979; Lima and Coimbra, 1986; Zhang et al., 1996; Han et al., 1998), especially in the primate spinal cord (Zhang and Craig, 1997). All labeled lamina I cells were counted in each of the sections; as before, cells were categorized as unclassified if they did not meet the criteria for the three main types, or if a portion of the soma and/or proximal dendritic tree was truncated by sectioning and precluded proper identification.

Corrections for double cell counts in the $40-\mu \mathrm{m}$-thick horizontal sections were not made because lamina I neurons are generally only $10-40 \mu \mathrm{m}$ in dorsoventral thickness, because they were classified and counted on the basis of somatal shape and primary dendritic origins rather than simply as labeled profiles, and because the relative proportions rather than the absolute numbers of the different cell types were of primary concern. Morphometric measurements were performed with an M4 image analysis system (Imaging Research, St. Catharines, Ontario, Canada).

Validation of the classification method. To ensure objective and consistent interpretation of the classification scheme, separate blind classifications were conducted, and an evaluation of inter-experimenter variability was performed. Independent counts of the same sets of sections were made by two groups of investigators (E.-T.Z. and A.D.C., and X.H.Y. and Y.D.), and the results were compared statistically. In addition, the counts in all double-labeled cases were performed by both teams.

Statistics. $\chi^{2}$ tests for contingency tables and ANOVA were used for comparison of differences in proportion of cells types. In cases in which values were expressed as mean \pm SEM, and in which percentages were used, the SEM was corrected for binomial distribution using an arcsine transform (Zar, 1984). The critical value for statistical significance was set at $p<0.05$. 


\section{RESULTS}

The appearance of NK-1r immunoreactivity in cervical and lumbar spinal cord was very similar to the distribution of NK-1r observed in the rat spinal cord using the same antiserum (Nakaya et al., 1994). NK-1r immunoreactivity was mostly restricted to lamina I of the monkey spinal cord, whereas virtually no staining was detected in lamina II (Fig. 1). A moderate level of staining was observed in laminae III-VI (Fig. 1). NK-1r immunoreactivity was mostly localized in neuronal cell bodies and dendrites. In transverse sections, most immunoreactive profiles were dendrites, but some cell bodies could also be identified in lamina I (Fig. 1). In horizontal sections, numerous NK1-r-IR cell bodies could be recognized (Fig. 2). Given the absence of NK-1r staining in lamina II, the cells identified in the most superficial horizontal sections unequivocally belonged to lamina I.

\section{Morphological types of NK-1r-IR cells}

Three major classes of cells were distinguished on the basis of cell body shape and proximal dendritic tree morphology: fusiform, pyramidal, and multipolar (Fig. 3). Fusiform cells had spindleshaped somata with elongated dendritic arbors extending from the two poles of the soma (Fig. 3, F1-F4). As described previously (Zhang et al., 1996; Zhang and Craig, 1997), the great majority of the fusiform cells were bipolar neurons with two major dendrites that extended in the rostrocaudal direction with little branching and little expansion in the mediolateral direction (Fig. 3, F3, F4). Some fusiform cells had one primary dendrite arising from each pole of the soma and gave rise to two or more dendrites directly from one of the poles (Fig. 3, F1, F2). Some of the fusiform cells were more irregular and had perikarya or dendrites oriented at various angles along the longitudinal and transverse axes. Pyramidal NK-1r-IR cells had pyramidal or triangular somata with a primary dendrite issuing from each corner of the soma (Fig. 3, P1, P2). Most of the pyramidal cells gave rise to one dendrite from each corner (Fig. 3, P1), whereas occasionally two or more dendrites arose from the same origin at one corner, and sometimes very fine dendrites arose from the side of the cell body (Fig. 3, P2). The pyramidal NK-1r-IR lamina I cells were large (Fig. 3, P1,P2), with an average length, width, and area of $40.5 \pm 2.1 \mu \mathrm{m}, 26 \pm 1.1 \mu \mathrm{m}$, and $1007 \pm 95.9 \mu \mathrm{m}^{2}$, respectively ( $n=25$, randomly selected from three monkeys). They were significantly larger than the average pyramidal lamina I STT neurons (length, $37.7 \pm 2.7 \mu \mathrm{m}$; width, $16.8 \pm 1.0 \mu \mathrm{m}$; area, $487.8 \pm 74.4 \mu \mathrm{m}^{2} ; n=16$ ) (Zhang and Craig, 1997). The multipolar NK-1r-IR cells included all four subtypes described in the classification of lamina I STT neurons in the monkey spinal cord (Zhang and Craig, 1997), including neurons with four dendrites (Fig. 3, M1), five or more dendrites (Fig. 3, M3), $\pi$-shaped (Fig. 3, M2), and tubular neurons (Fig. 3, M4).

Neurons that differed in morphology and that could not be categorized into any of the above three types of neurons were counted as unclassified. These included cells that had transitional shapes (Fig. 4A,C), cells with small round or oval, unipolar perikarya, and cells whose shape could not be recognized because the soma had been divided by sectioning. The dendrites of unclassified cells extended in any direction within lamina I (Fig. $4 B, D)$.

\section{Distribution of NK-1r immunoreactivity with respect to the morphological types of lamina I cells}

To identify which morphological types of lamina I neurons express NK-1r, we counted labeled cells in horizontal sections of the cervical and lumbar enlargements of four adult macaque monkeys. First, to ensure consistent classification, labeled cells were counted independently by the two teams of observers in this study. For example, in one set of sections, a total of 206 NK-1r-IR lamina I neurons was categorized (one side of the dorsal horn only) by one team as 82 fusiform, 7 pyramidal, 79 multipolar, and 38 unclassified, and in the same sections a total of 195 labeled neurons was identified by the other team and classified as 85 fusiform, 10 pyramidal, 67 multipolar, and 33 unclassified. The small differences could be accounted for by differences between observers in the threshold for the identification of a positively labeled cell and in the categorization of cells with transitional shapes. There was no significant difference $\left(\chi^{2}\right.$ contingency test; $p>0.2)$ between the two teams of experimenters with respect to the relative distribution of NK-1r-IR lamina I cell types. Thus, it was considered that the classification scheme was sufficiently objective and consistent to obtain reproducible and reliable results.

A total of 2924 NK-1r-IR cells in lamina I were classified in the four monkeys. Of these neurons, there were on average $51 \%$ fusiform, $6 \%$ pyramidal, and $33 \%$ multipolar cells in C7-8 segments and $31 \%$ fusiform, $6 \%$ pyramidal, and $53 \%$ multipolar cells in L6-7 segments. The remaining neurons were unclassified. Cervical segments contained more NK-1r-IR fusiform than multipolar cells, and lumbar segments contained more NK-1r-IR multipolar than fusiform cells (Table 1).

\section{Distribution of NK-1r immunoreactivity among spinothalamic lamina I neurons}

To further characterize the population of NK-1r-IR neurons, we performed double-labeling experiments on retrogradely $\mathrm{CTb}$ labeled lamina I STT cells in three monkeys. The thalamic injections were comparable to those in a previous report (Zhang and Craig, 1997) and covered all the medial and lateral thalamus with no spread to the hypothalamus or mesencephalon. A total of 1117 spinal lamina I STT neurons were counted on the side contralateral to thalamic injections in spinal cord segments C6-7 and L6-7. Of the labeled STT neurons, 693 (62\%) stained positive for NK-1r. The photomicrographs in Figure 5 show examples of double-labeled neurons immunoreactive for both $\mathrm{CTb}$ and NK-1r along with other neurons that stained positive only for $\mathrm{CTb}$ or NK-1r. The immunoreactive labeling for retrogradely transported $\mathrm{CTb}$ was distributed intracellularly throughout the soma and dendrites, which differed from the typical appearance of NK-1r-IR labeling along the plasma membrane of the neurons (Fig. 6). Thus, identification of double-labeled neurons was unequivocal.

The distribution of retrogradely labeled lamina I STT neurons with respect to the different morphological categories is shown in Figure 7. This distribution was comparable to that previously reported for the cervical and lumbar enlargements (Zhang and Craig, 1997). Pyramidal neurons represented $\sim 25 \%$ of the population of lamina I STT cells in both areas combined, whereas fusiform neurons represented $39 \%$ and multipolar 29\%; the remainder were unclassified. This contrasts strongly with the distribution of cell types among lamina I neurons that were NK-1r-IR for the three monkeys in which double immunofluorescence labeling was performed (Fig. 7). The relative proportion of pyramidal NK-1r-IR cells was significantly lower than the proportion of pyramidal cells among lamina I STT neurons $(p<$ 0.01 ). The relative distribution of each cell type among lamina I STT cells that were double-labeled for NK-1r-IR similarly differed from 

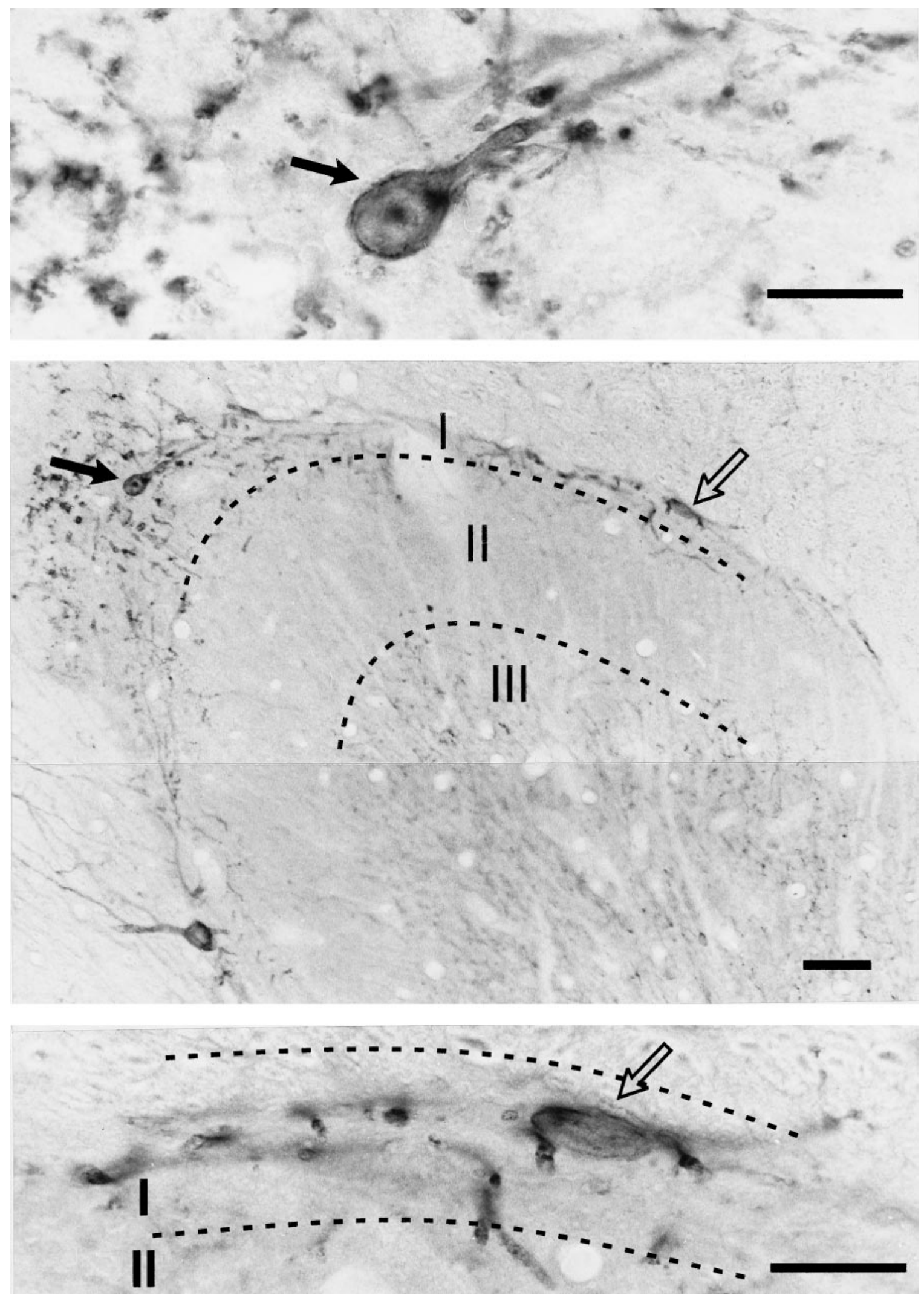

Figure 1. Photomicrograph of NK-1r immunoreactivity in the lumbar spinal cord in a transverse section. Laminae are indicated with roman numerals. Most NK-1r immunoreactivity was located in lamina I with moderate staining in laminae III-IV. The photomicrographs at the top and bottom are enlargements of the neurons at the locations indicated by the arrows. Scale bars: top and bottom, $50 \mu \mathrm{m} ;$ middle, $100 \mu \mathrm{m}$. 


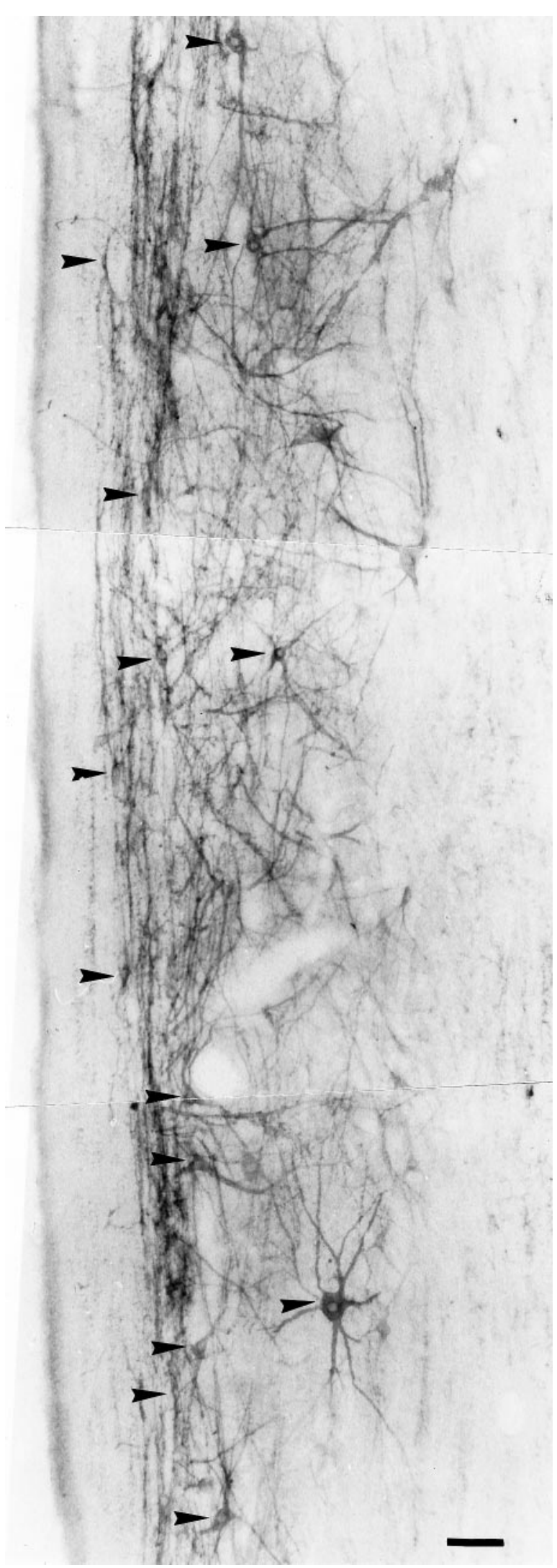

Figure 2. Photomicrograph of NK-1r immunoreactivity in a 40- $\mu$ m-thick horizontal section of the lumbar spinal cord. The perikarya and the proximal dendrites were well stained. Their primary orientation in this plane can be observed. This plane of section is preferred for morpholog- the overall distribution among all lamina I STT cells (on average for both cervical and lumbar segments: fusiform $50 \%$, pyramidal $10 \%$, multipolar 33\%, the remainder being unclassified; Fig. 7). Mainly, the proportion of NK-1r-IR pyramidal cells among lamina I STT neurons was significantly lower than the proportion of this cell type among all lamina I STT neurons (Fig. 7).

To further examine the under representation of NK-1r-IR cells among pyramidal neurons, we calculated the proportion of lamina I STT neurons that were immunoreactive for NK-1r within each morphological category (Table 2). Overall, 62\% of all lamina I STT neurons were immunoreactive for NK-1r in the cervical and lumbar enlargements combined. This proportion rose to $81 \%$ for the fusiform lamina I STT cells and $71 \%$ for the multipolar lamina I STT cells, whereas only $25 \%$ of the pyramidal lamina I STT cells were immunoreactive for NK-1r. Thus, the majority of lamina I STT cells found to express NK-1r were fusiform and multipolar cells, and conversely, most pyramidal and some fusiform and multipolar lamina I STT cells did not express NK-1r.

Finally, of all of the NK-1r-immunostained lamina I neurons in the cervical and lumbar enlargements, $69 \%$ were also immunoreactive for $\mathrm{CTb}$. This indicates that the majority, but not all, of the NK-1r-IR lamina I cells contributed to the spinothalamic tract. In addition, considering that $62 \%$ of lamina I STT neurons were NK-1r-IR and the recent estimate that $\sim 45 \%$ of all lamina I neurons are NK-1r-IR in the rat (Todd et al., 1998), our results suggest that the proportion of lamina I neurons that project to the thalamus in the spinal enlargements of the monkey may be $\sim 50 \%$ $(\mathrm{NK}-1 \mathrm{r}-\mathrm{IR}$ and STT $=0.45 \times 0.69 ; \mathrm{STT}$, non-NK-1r-IR $=0.45 \times$ $0.69 \times 0.38 / 0.62)$.

\section{DISCUSSION}

Our results indicate that lamina I neurons immunoreactive for the NK-1 receptor can be classified into three major morphological categories, fusiform, pyramidal, and multipolar, in the macaque spinal cord based on somatal shape and primary dendrite orientation, as had been described earlier for lamina I STT neurons in the monkey (Zhang and Craig, 1997). The main observation of this study was that two of these morphological types, the fusiform and multipolar cells, stain positive for NK-1r, whereas only a small proportion of pyramidal cells do. These results are of particular interest in view of the correlation recently observed in the cat between these three morphological types and the three main functional classes of lamina I neurons (Han et al., 1998).

\section{NK-1r immunoreactivity is densest in lamina I}

Our present results are in general agreement with the distribution of NK-1r immunoreactivity described in the rat spinal cord applying the antibody used in this study. In the rat, NK-1r immunoreactivity was densest in lamina I, sparse in lamina II, and moderate in laminae III-VI (Nakaya et al., 1994). In the monkey spinal cord, even less staining was observed in lamina II, a result that is consistent with the distribution of NK-1r immunoreactivity reported in the rat spinal cord using this (Li et al., 1996, 1997) and another antibody against the NK-1r (Liu et al., 1994; Brown et al., 1995; Littlewood et al., 1995; Marshall et al., 1996).

ical classification of lamina I neurons because their perikarya and dendrites are mainly oriented in the rostrocaudal and mediolateral axes. Several cell bodies can be distinguished (arrows). Lateral part is left and rostral part is up. Scale bar, $100 \mu \mathrm{m}$. 

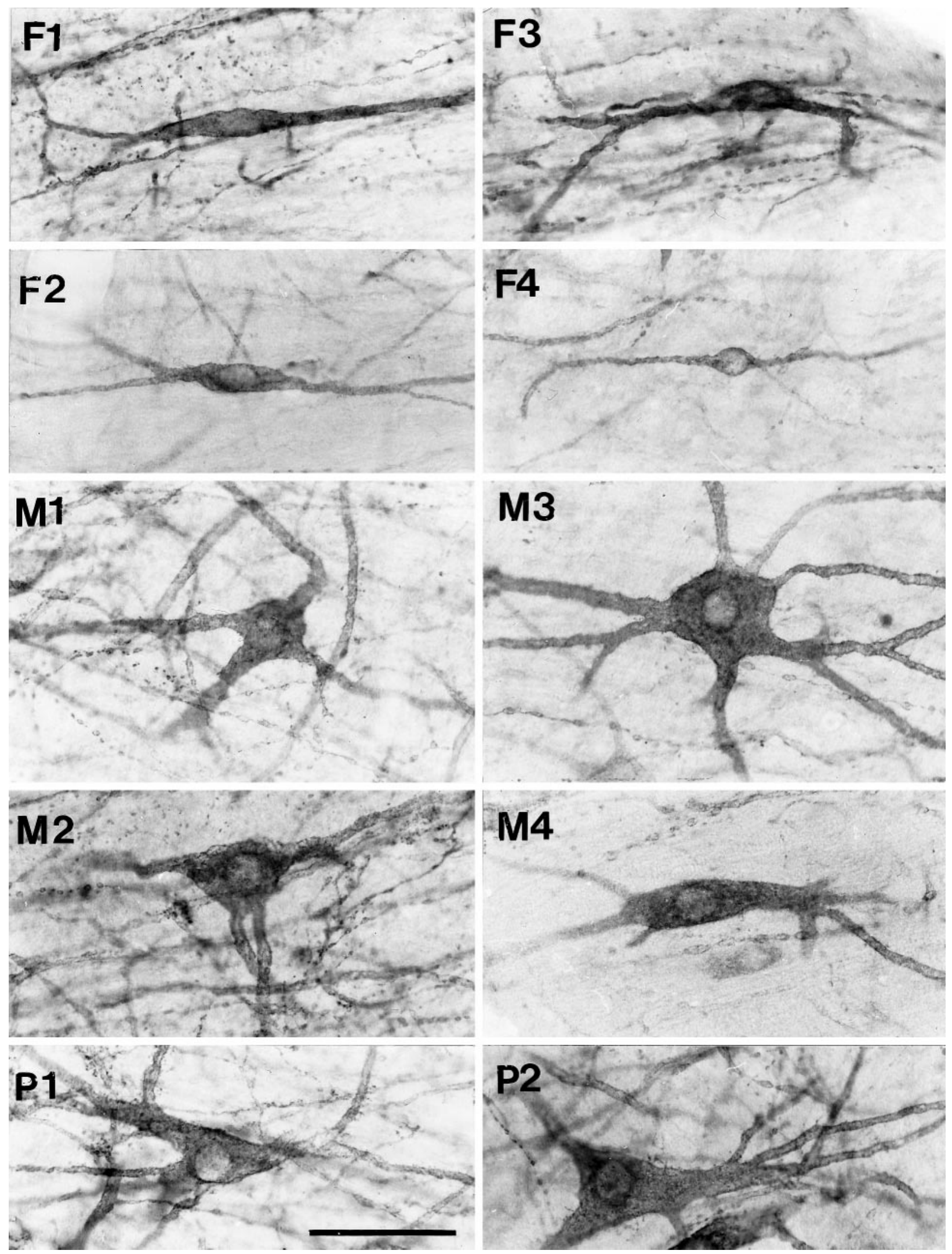

Figure 3. Photomicrographs showing examples of the three main types of NK-1r-IR lamina I neurons in 40- $\mu$ m-thick horizontal sections: fusiform $(F)$ cells with spindle-shaped somata and two main longitudinal dendritic arbors; pyramidal $(P)$ cells with triangular somata and three main dendrites oriented primarily longitudinally; multipolar $(M)$ cells with polygonal somata and four or more dendrites directed longitudinally and mediolaterally. Scale bar, $100 \mu \mathrm{m}$. 

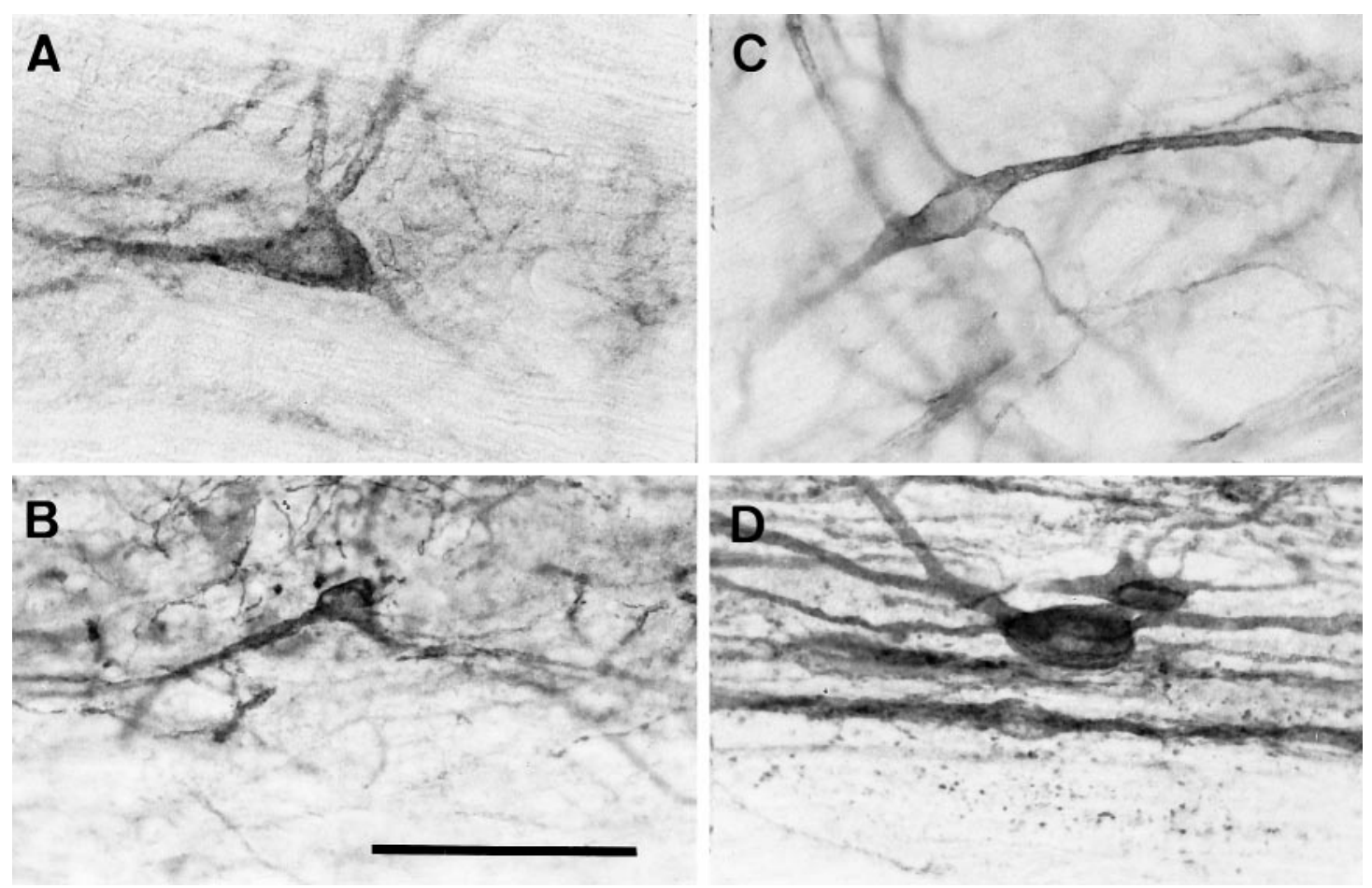

Figure 4. Photomicrographs showing examples of unclassified NK-1r-IR neurons in horizontal sections. $A$, $C$, Neurons with transitional shapes $(A$, a pyramidal soma with four dendrites; $C$, a fusiform soma and several dendrites). $B, D$, Cells with small round unipolar perikarya, the dendrites of which extended in different directions in lamina I. Scale bar, $100 \mu \mathrm{m}$.

Table 1. Relative distribution of lamina I cell types among NK-1r-IR cells in the cervical (C) and lumbar (L) enlargements

\begin{tabular}{lllllll} 
Case & Segment & $\mathrm{F}(\%)$ & $\mathrm{P}(\%)$ & $\mathrm{M}(\%)$ & $\mathrm{U}(\%)$ & $\begin{array}{l}\text { Total } \\
\text { number }\end{array}$ \\
\hline M03 & $\mathrm{C}$ & 46.5 & 4.9 & 35.1 & 13.5 & 385 \\
M04 & $\mathrm{C}$ & 50.1 & 5.8 & 34.0 & 10.0 & 379 \\
M05 & $\mathrm{C}$ & 56.8 & 6.3 & 27.9 & 9.0 & 333 \\
M06 & $\mathrm{C}$ & 51.8 & 6.2 & 36.9 & 5.1 & 390 \\
Mean & & $\mathbf{5 1}$ & $\mathbf{6}$ & $\mathbf{3 4}$ & $\mathbf{9}$ & $\mathbf{3 7 2}$ \\
M03 & L & 32.9 & 6.6 & 49.4 & & \\
M04 & L & 30.1 & 5.4 & 55.7 & 8.7 & 395 \\
M05 & L & 33.4 & 5.6 & 50.8 & 10.1 & 332 \\
M06 & L & 29.9 & 7.3 & 56.2 & 6.5 & 354 \\
Mean & & $\mathbf{3 2}$ & $\mathbf{6}$ & $\mathbf{5 3}$ & $\mathbf{9}$ & $\mathbf{3 5 9}$ \\
\hline
\end{tabular}

F, Fusiform cells; P, pyramidal cells; M, multipolar cells; U, unclassified cells.

\section{Morphological types of NK-1r-IR cells}

The approach used in the current study allowed a classification of lamina I neurons into three general types. We did not attempt a further subdivision of the cells of each type as in other studies (Gobel, 1978; Lima and Coimbra, 1986; Zhang et al., 1996; Zhang and Craig, 1997), although subtypes of each were noted. The general categorization into three basic cell types represents a scheme that is consistent across species (Gobel, 1978; Willis et al., 1979; Lima and Coimbra, 1986; Zhang et al., 1996; Z hang and Craig, 1997), and it proved to be reliable enough to minimize variability in interpretation of classification criteria between observers. As previously shown, these cell types cannot be properly identified in transverse sections (Gobel, 1978; Lima and Coimbra, 1986; Zhang et al., 1996; Zhang and Craig, 1997). Although additional information can be obtained in sagittal sections regarding cells with ventrally directed dendrites in the rat (Lima and Coimbra, 1986), the basic morphological types are best identified in horizontal sections because lamina I cells arborize primarily in this plane (Gobel, 1978; Light et al., 1979, 1993; Steedman et al., 1985; Hylden et al., 1986; Lima and Coimbra, 1986; Zhang et al., 1996; Zhang and Craig, 1997; Han et al., 1998).

Previous studies have reported NK-1r labeling in morphologically identified (Brown et al., 1995) and retrogradely labeled STT cells in lamina I (Marshall et al., 1996) of the rat. Brown et al. (1995) reported numerous fusiform neurons immunoreactive for NK-1r. However, neither study attempted further classification of the cells.

Fusiform, pyramidal, and multipolar cells are relatively uni- 

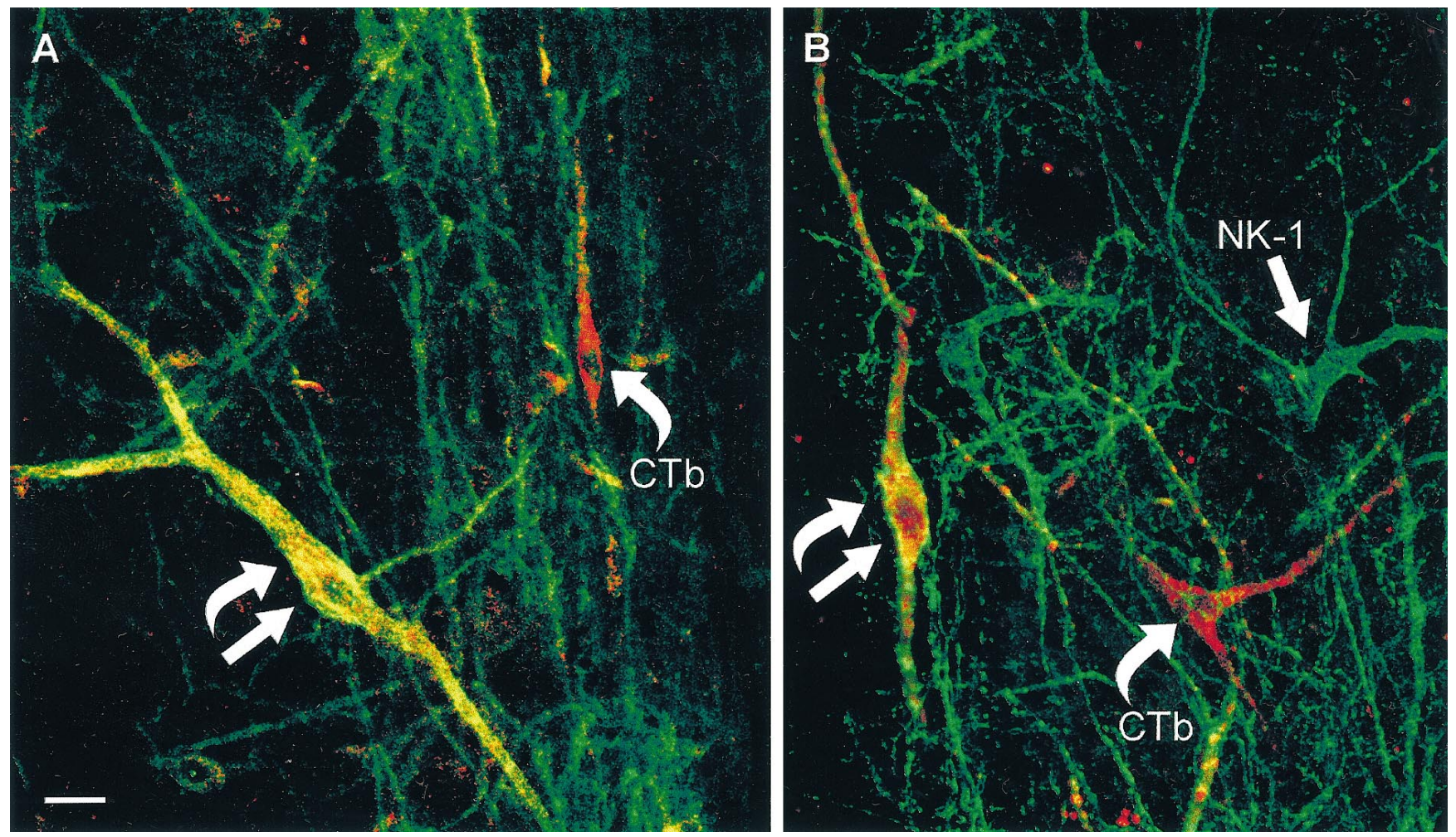

Figure 5. Confocal micrographs of lamina I neurons illustrating the pattern of labeling for NK-1r and retrograde CTb. The micrographs represent reconstructions from 20 serial optical sections (1.0 $\mu \mathrm{m}$ apart) in $A$ and seven in $B$. The red color represents immunoreactivity for CTb (curved arrows), and the green corresponds to NK-1r immunoreactivity (straight arrows). The two signals were superimposed so that double-labeled structures appear in yellow (double arrows). Note in $A$, the double-labeled (STT + NK-1r) neuron on the left that contrasts with the CTb-only labeled fusiform neuron on the right. Therefore, the latter STT fusiform cell is NK-1r-immunonegative. In $B$, there is one fusiform cell that was double-labeled, whereas a $\mathrm{CTb}$-labeled pyramidal neuron was not NK-1r-IR, and a multipolar neuron stained positive for NK-1r, but not for CTb. Scale bar, $50 \mu \mathrm{m}$.
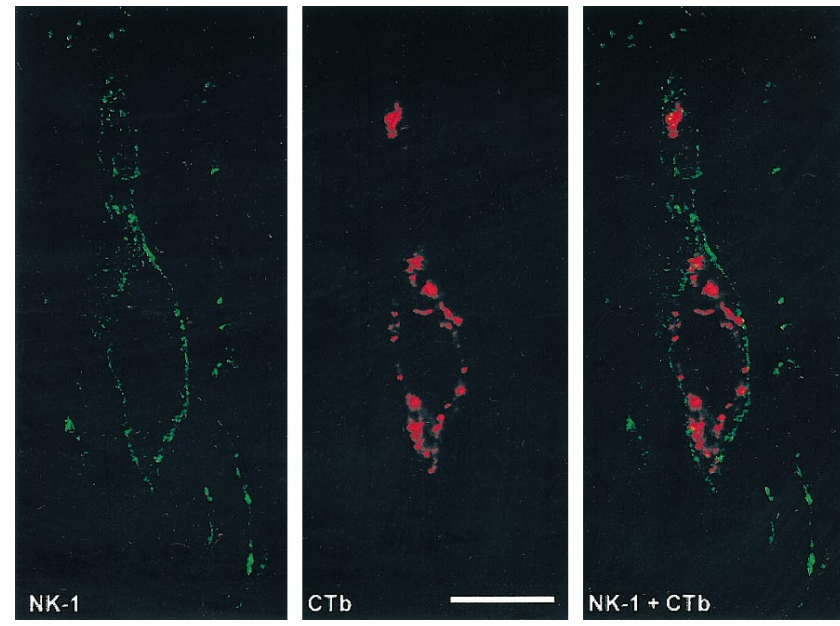

Figure 6. Confocal micrograph illustrating a reconstruction from four serial optical sections $(0.5 \mu \mathrm{m}$ apart $)$ to indicate the difference in the nature of labeling obtained with the retrogradely transported $\mathrm{CTb}$ (mid$d l e)$ versus that of NK-1r immunoreactivity (left). The cytoplasmic pattern of labeling obtained with $\mathrm{CTb}$ could not be confused with the typically plasma membrane-associated pattern of NK-1r-IR staining. The micrograph on the right is a superimposition of the two signals. Scale bar, $25 \mu \mathrm{m}$.

formly distributed in the lumbar and cervical enlargements of the spinal cord. Consistent with previous studies in cat and monkey (Zhang et al., 1996; Zhang and Craig, 1997), the proportions of lamina I STT cell types in the cervical enlargement were: fusi- form $42 \%$, pyramidal $28 \%$, multipolar $26 \%$, with the remainder unclassified. In their Golgi study in the rat cervical spinal cord, Lima and Coimbra (1986) classified lamina I cells as fusiform, multipolar, flattened, and pyramidal, but grouping their multipolar and flattened cell types together (as these two categories would fall under the multipolar type in the present study), their distribution of Golgi-stained cell types would similarly be fusiform $39 \%$, pyramidal $36 \%$, and multipolar $25 \%$. Thus, based on retrograde labeling from the thalamus in cat and monkey or on random Golgi sampling in the rat, the proportion of pyramidalshaped lamina I neurons is $>25 \%$. This contrasts strongly with the present finding that only $4-7 \%$ of NK-1r-IR lamina I neurons were pyramidal. Our results show that only a small proportion of pyramidal neurons express NK-1r. Furthermore, the pyramidal lamina I neurons that were NK-1r-IR may be a derivative subset, because they were significantly larger than the average lamina I STT pyramidal neurons (Zhang and Craig, 1997).

\section{Functional relevance}

The differentiation of lamina I cells into separate morphological types, which also differ in phenotype, raises the possibility of a correspondence with function. Indeed, recent evidence indicating a direct correspondence between the morphological characteristics and functional properties of lamina I neurons in the cat (Han et al., 1998) is consistent with the view that there are three basic types of lamina I neuron: fusiform NS cells, pyramidal COLD cells, and multipolar HPC cells. The possibility that this correspondence is valid as well in the monkey is supported by a recent finding that a distinct subregion of trigeminal lamina $\mathrm{I}$ in the owl 

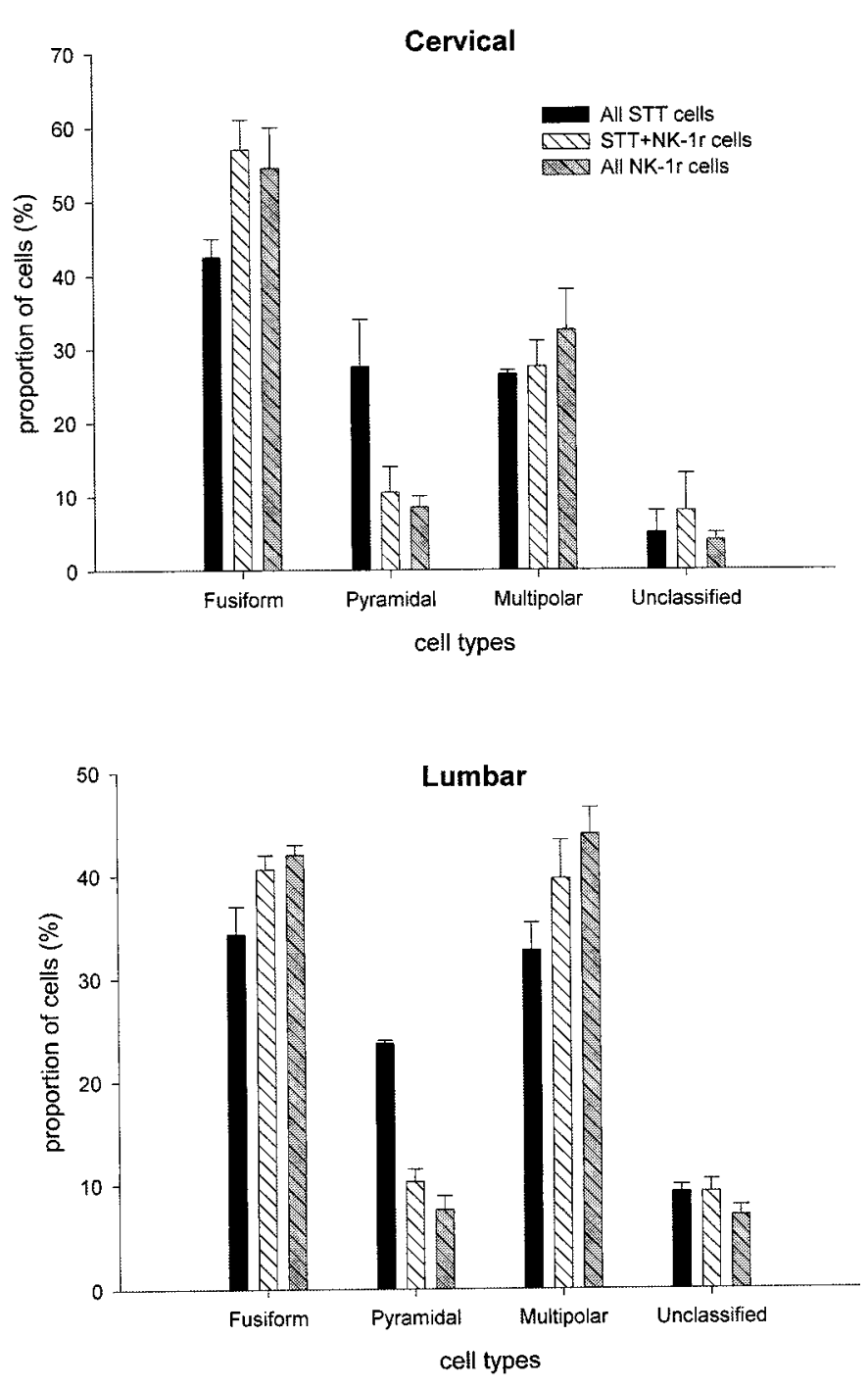

Figure 7. Comparison of the relative distribution of morphological types of cells among all lamina I neurons that were immunoreactive for NK-1r (All NK-1r), all that were retrogradely labeled with CTb injected in the thalamus (All STT), and those that were both NK-1r-IR and retrogradely CTb-labeled $(S T T+N K-1 r)$. The vertical bars represent the means \pm SEM (after correction, see Material and Methods) from the three monkeys in which double immunofluorescence labeling was performed for the detection of both NK-1r and CTb immunoreactivity. The proportion of pyramidal cells among NK-1r-IR neurons was significantly lower $(p<$ 0.01 ) than that observed among all STT cells.

$\begin{aligned} & \text { Table 2. Average proportions of lamina I STT neurons within each } \\
& \text { morphological category that were immunoreactive for NK-1r in the } \\
& \text { cervical (C; C6-7) and lumbar (L; L6-7) enlargements of three } \\
& \text { monkeys }\end{aligned}$
\begin{tabular}{lllll} 
Segment & F (\%) & P (\%) & M (\%) & U (\%) \\
\hline C & $80.5 \pm 5.5$ & $22.0 \pm 4.0$ & $64.0 \pm 13.0$ & $77.5 \pm 22.5$ \\
L & $81.3 \pm 7.3$ & $29.3 \pm 3.8$ & $81.0 \pm 4.0$ & $67.7 \pm 10.7$
\end{tabular}

F, Fusiform cells; P, pyramidal cells; M, multipolar cells; U, unclassified cells.

monkey, in which clusters of thermoreceptive-specific COLD cells can be recorded, contains almost exclusively pyramidalshaped neurons (Craig et al., 1999). These results indicate that pyramidal neurons represent a non-nociceptive type of neuron, whereas multipolar and fusiform lamina I cells are nociceptive. The previous finding that SP only excites nociceptive neurons (Henry, 1976; Randic and Miletic, 1977; Salter and Henry, 1991) suggests that non-nociceptive neurons do not express NK-1r. Thus, the fact that a minority of pyramidal neurons were found to express NK-1r in the present study reinforces the idea that SP is specifically associated with nociception and further indicates a correspondence between the structural and functional specialization of lamina I neurons.

It is also interesting to note that whereas non-nociceptive cells do not seem to express NK-1r (this study and Henry, 1976; Randic and Miletic, 1977; Salter and Henry, 1991), they also do not appear to receive direct SP input in significant amount. This contrasts with the observation that SP-responsive nociceptive neurons receive abundant SP synaptic inputs (De Koninck et al., 1992; Ma et al., 1996). Yet, other studies have shown a mismatch between the distribution of SP immunoreactivity and NK-1r-IR in some regions of the CNS (Nakaya et al., 1994; Brown et al., 1995), raising the question of whether SP must diff use away from its release site to reach its receptor. The expression of NK-1r by the postsynaptic cell may therefore be what confers specificity to the signal. Several lines of evidence suggest however that, at the cellular level, in areas where the transmitter and the receptor are both present, they are in close association. A recent confocal microscopy study indicated that neurons in laminae III-IV with NK-1r-IR dendritic branches in laminae I/II receive abundant innervation from SP-IR axons, in contrast to neighboring cholinergic neurons (Naim et al., 1997). Similarly, the subregions of lamina I in the owl monkey that contain clusters of innocuous thermoreceptive pyramidal neurons (Craig et al., 1999) are characteristically devoid of SP-IR fibers, consistent with the fact the innocuous cooling afferents do not express SP (Lawson et al., 1997) and that only a small proportion of lamina I pyramidal cells appear to express NK-1r (present study). Finally, quantification at the ultrastructural level in the superficial dorsal horn shows that SP-IR boutons preferentially innervate NK-1r-IR dendritic profiles (McLeod et al., 1998). The latter findings suggest specialization in both receptor expression and axon targeting for this spinal peptidergic system. Whether this means that SP acts always at a short distance from its release site remains to be demonstrated directly. In this case, the apparent discrepancy with the mismatch found in some regions would have to indicate that, in areas where SP fibers are present without the NK-1r, these correspond to en passant fibers or collaterals that do not participate in SP signaling [e.g., glutamate may be the main active transmitter at these synapses (De Biasi and Rustioni, 1988)].

\section{Subpopulation of NK-1r-IR cells among spinothalamic tract neurons in lamina I}

Approximately $62 \%$ of the lamina I STT neurons were immunoreactive for NK-1r in the present study. This result is similar to the 77\% observed for STT cells in lamina I of the rat spinal cord (Marshall et al., 1996). The proportion increases to $77 \%$ in the current study when considering only fusiform and multipolar cells. Indeed, fusiform and multipolar represented most of the NK-1r-positive STT cells, whereas only $10 \%$ of these were pyramidal.

Our results also indicate that a subpopulation of lamina I STT cells do not express NK-1r. Aside from the pyramidal cells, a significant proportion (25\%) of fusiform and multipolar neurons do not express NK-1r. Given that Han et al. (1998) reported that all fusiform and multipolar neurons are nociceptive, this result 
indicates that a subpopulation of lamina I STT nociceptive cells do not respond to SP. This observation is consistent with the finding that, throughout the dorsal horn, SP does not excite all nociceptive neurons, but rather, according to Salter and Henry (1991), only $\sim 75 \%$. Furthermore, there is similar evidence suggesting that many nociceptive primary afferent fibers do not contain SP (Lawson et al., 1997). This result raises the question of whether pathological conditions known to alter NK-1r expression (Abbadie et al., 1996) may result in shifts in the population of neurons that express this receptor.

\section{Conclusion}

Our results show that the substance $\mathrm{P}$ receptor $(\mathrm{NK}-1 \mathrm{r})$ is differentially expressed among morphologically defined cell groups in lamina I of the primate spinal dorsal horn. Given the association of these morphological features with particular functional properties of lamina I neurons and the fact that substance P only acts on selected functional classes of neurons, our results provide convergent evidence of a differentiation of cells in this area based on their structural, functional, and pharmacological characteristics. These results will also prove particularly useful as a tool for the definition of neuronal populations in anatomical studies and in those using spinal cord slice preparations.

\section{REFERENCES}

Abbadie C, Brown JL, Mantyh PW, Basbaum AI (1996) Spinal cord substance $\mathrm{P}$ receptor immunoreactivity increases in both inflammatory and nerve injury models of persistent pain. Neuroscience 70:201-209.

Beal JA, Penny JE, Bicknell HR (1981) Structural diversity of marginal (lamina I) neurons in the adult monkey (Macaca mulatta) lumbosacral spinal cord: a Golgi study. J Comp Neurol 202:237-254.

Brown JL, Liu H, Maggio JE, Vigna SR, Mantyh PW, Basbaum AI (1995) Morphological characterization of substance P receptorimmunoreactive neurons in the rat spinal cord and trigeminal nucleus caudalis. J Comp Neurol 356:327-344.

Craig AD (1996a) An ascending general homeostatic afferent pathway originating in lamina I. Prog Brain Res 107:225-242.

Craig AD (1996b) Pain, temperature, and the sense of the body. In: Somesthesis and the neurobiology of the somatosensory cortex (Franzen O, Johansson RS, Terenius L, eds), pp 27-39. Basel: Birkhauser.

Craig AD, Bushnell MC (1994) The thermal grill illusion: unmasking the burn of cold pain. Science 265:252-255.

Craig AD, Kniffki KD (1985) Spinothalamic lumbosacral lamina I cells responsive to skin and muscle stimulation in the cat. J Physiol (Lond) 365:197-221.

Craig AD, Serrano LP (1994) Effects of systemic morphine on lamina I spinothalamic tract neurons in the cat. Brain Res 636:233-244.

Craig AD, Bushnell MC, Zhang ET, Blomqvist A (1994) A thalamic nucleus specific for pain and temperature sensation. Nature 372:770-773.

Craig AD, Zhang ET, Blomqvist A (1999) A distinct thermoreceptive sub-region of lamina I in nucleus caudalis of the owl monkey. J Comp Neurol 404:221-234.

Cuello AC (1987) Peptides as neuromodulators in primary sensory neurons. Neuropharmacology 26:971-979.

De Biasi S, Rustioni A (1988) Glutamate and substance P coexist in primary afferent terminals in the superficial laminae of spinal cord. Proc Natl Acad Sci USA 85:7820-7824.

De Koninck Y, Henry JL (1991) Substance P-mediated slow excitatory postsynaptic potential in vivo in dorsal horn neurons elicited by noxious stimulation. Proc Natl Acad Sci USA 88:11344-11348.

De Koninck Y, Ribeiro-da-Silva A, Henry JL, Cuello AC (1992) Spinal neurons exhibiting a specific nociceptive response receive abundant substance P-containing synaptic contacts. Proc Natl Acad Sci USA 89:5073-5077.

Dostrovsky JO, Craig AD (1996) Cooling-specific spinothalamic neurons in the monkey. J Neurophysiol 76:3656-3665.

Ericson H, Blomqvist A (1988) Tracing of neuronal connections with cholera toxin subunit B: light and electron microscopic immunohisto- chemistry using monoclonal antibodies. J Neurosci Methods 24:225-235.

Gobel S (1978) Golgi studies of the neurons in layer I of the dorsal horn of the medulla (trigeminal nucleus caudalis). J Comp Neurol 180:375-393.

Han ZS, Zhang ET, Craig AD (1998) Nociceptive and thermoreceptive lamina I neurons are anatomically distinct. Nat Neurosci 1:218-225.

Henry JL (1976) Effects of substance P on functionally identified units in cat spinal cord. Brain Res 114:439-451.

Henry JL (1982) Relation of substance P to pain transmission: neurophysiological evidence. Ciba Found Symp 9:206-224.

Hylden JL, Hayashi H, Dubner R, Bennett GJ (1986) Physiology and morphology of the lamina I spinomesencephalic projection. J Comp Neurol 247:505-515.

Lawson SN, Crepps BA, Perl ER (1997) Relationship of substance P to afferent characteristics of dorsal root ganglion neurones in guinea-pig. J Physiol (Lond) 505:177-191.

Li JL, Ding YQ, Shigemoto R, Mizuno N (1996) Distribution of trigeminothalamic and spinothalamic-tract neurons showing substance $\mathrm{P}$ receptor-like immunoreactivity in the rat. Brain Res 719:207-212.

Li JL, Kaneko T, Shigemoto R, Mizuno N (1997) Distribution of trigeminohypothalamic and spinohypothalamic tract neurons displaying substance $\mathrm{P}$ receptor-like immunoreactivity in the rat. J Comp Neurol 378:508-521.

Light AR (1992) The initial processing of pain and its descending control: spinal and trigeminal systems. Basel: Karger.

Light AR, Trevino DL, Perl ER (1979) Morphological features of functionally defined neurons in the marginal zone and substantia gelatinosa of the spinal dorsal horn. J Comp Neurol 186:151-171.

Light AR, Sedivec MJ, Casale EJ, Jones SL (1993) Physiological and morphological characteristics of spinal neurons projecting to the parabrachial region of the cat. Somatosens Mot Res 10:309-325.

Lima D, Coimbra A (1986) A Golgi study of the neuronal population of the marginal zone (lamina I) of the rat spinal cord. J Comp Neurol 244:53-71.

Littlewood NK, Todd AJ, Spike RC, Watt C, Shehab SA (1995) The types of neuron in spinal dorsal horn which possess neurokinin-1 receptors. Neuroscience 66:597-608.

Liu H, Brown JL, Jasmin L, Maggio JE, Vigna SR, Mantyh PW, Basbaum AI (1994) Synaptic relationship between substance P and the substance $\mathrm{P}$ receptor: light and electron microscopic characterization of the mismatch between neuropeptides and their receptors. Proc Natl Acad Sci USA 91:1009-1013.

Ma W, Ribeiro-da-Silva A, De Koninck Y, Radhakrishnan V, Henry JL, Cuello AC (1996) Quantitative analysis of substance P immunoreactive boutons on physiologically characterized dorsal horn neurons in the cat lumbar spinal cord. J Comp Neurol 376:45-64.

Marshall GE, Shehab SA, Spike RC, Todd AJ (1996) Neurokinin-1 receptors on lumbar spinothalamic neurons in the rat. Neuroscience 72:255-263.

McLeod AL, Krause JE, Cuello AC, Ribeiro-da-Silva A (1998) Preferential synaptic relationships between substance P-immunoreactive boutons and neurokinin-1 receptor sites in the rat spinal cord. Proc Natl Acad Sci USA 95:15775-15780.

Naim M, Spike RC, Watt C, Shehab SA, Todd AJ (1997) Cells in laminae III and IV of the rat spinal cord that possess the neurokinin-1 receptor and have dorsally directed dendrites receive a major synaptic input from tachykinin-containing primary afferents. J Neurosci 17:5536-5548.

Nakaya Y, Kaneko T, Shigemoto R, Nakanishi S, Mizuno N (1994) Immunohistochemical localization of substance $\mathrm{P}$ receptor in the central nervous system of the adult rat. J Comp Neurol 347:249-274.

Otsuka M, Yanagisawa M (1990) Pain and neurotransmitters. Cell Mol Neurobiol 10:293-302.

Perl ER (1984) Pain and nociception. In: Sensory processes (DarianSmith I, ed), pp 915-975. Bethesda: American Physiological Society.

Priestley JV, Cuello AC (1989) Ultrastructural and neurochemical analysis of synaptic input to trigemino-thalamic projection neurones in lamina I of the rat: a combined immunocytochemical and retrograde labelling study. J Comp Neurol 285:467-486.

Randic M, Miletic V (1977) Effect of substance P in cat dorsal horn neurones activated by noxious stimuli. Brain Res 128:164-169.

Rexed B (1952) The cytoarchitectonic organization of the spinal cord in the cat. J Comp Neurol 96:415-495. 
Ribeiro-da-Silva A, Cuello AC (1995) Organization of peptidergic neurons in the dorsal horn of the spinal cord: anatomical and functional correlates. Prog Brain Res 104:41-59.

Salter MW, Henry JL (1991) Responses of functionally identified neurones in the dorsal horn of the cat spinal cord to substance P, neurokinin A and physalaemin. Neuroscience 43:601-610.

Shigemoto R, Nakaya Y, Nomura S, Ogawa-Meguro R, Ohishi H, Kaneko T, Nakanishi S, Mizuno N (1993) Immunocytochemical localization of rat substance P receptor in the striatum. Neurosci Lett 153:157-160.

Steedman WM, Molony V, Iggo A (1985) Nociceptive neurones in the superficial dorsal horn of cat lumbar spinal cord and their primary afferent inputs. Exp Brain Res 58:171-182.
Todd AJ, Spike RC, Polgár E (1998) A quantitative study of neurons which express neurokinin-1 and somatostatin sst2a receptor in rat spinal dorsal horn. Neuroscience 85:459-473.

Willis WD (1985) The pain system: the neural basis of nociceptive transmission in the mammalian nervous system. Basel: Karger.

Willis WD, Kenshalo Jr DR, Leonard RB (1979) The cells of origin of the primate spinothalamic tract. J Comp Neurol 188:543-573.

Zar JH (1984) Biosatistical analysis. Englewood Cliffs, NJ: Prentice-Hall.

Zhang ET, Craig AD (1997) Morphology and distribution of spinothalamic lamina I neurons in the monkey. J Neurosci 17:3274-3284.

Zhang ET, Han ZS, Craig AD (1996) Morphological classes of spinothalamic lamina I neurons in the cat. J Comp Neurol 367:537-549. 